\title{
Cell-Free Mitochondrial DNA in Embryo Spent Culture Medium as Biomarker for In- Vitro Fertilization Outcome and its Potential Clinical Use
}

\section{ISSN: 2640-9666}

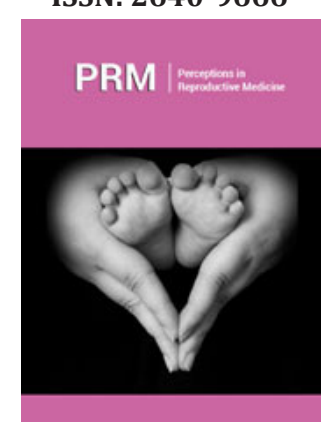

*Corresponding author: Scaruffi P, UOS Physiopathology of Human Reproduction, IRCCS Ospedale Policlinico San Martino, Genova, Italy

Submission: 进 March 5, 2021

Published: 眥 March 10, 2021

Volume 4 - Issue 4

How to cite this article: Scaruffi $P$, Stiglian S, Anserini P. Cell-Free Mitochondrial DNA in Embryo Spent Culture Medium as Biomarker for In-Vitro Fertilization Outcome and its Potential Clinical Use. Perceptions in Reproductive Medicine. 4(3). PRM. 000591. 2021.

DOI: 10.31031/PRM.2021.04.000591

Copyright@ Scaruffi P, This article is distributed under the terms of the Creative Commons Attribution 4.0 International License, which permits unrestricted use and redistribution provided that the original author and source are credited.

\section{Scaruffi P*, Stigliani S and Anserini P}

UOS Physiopathology of Human Reproduction, IRCCS Ospedale Policlinico San Martino, Genova, Italy

Opinion

On 2013 for the first time, we discovered both cell-free genomic DNA (cf-gDNA) and cellfree mithocondrial DNA (cf-mtDNA) in spent culture media of human cleavage-stage embryos [1]. Analysis of DNA profiles of day 3 spent media demonstrated that cf-mtDNA/cf-gDNA ratio was correlated with embryo quality, and in particular it was significantly associated with degree of embryo fragmentation. Based on our results, the higher cf-mtDNA/cf-gDNA ratio was associated with blastocyst's developmental competence, trophoectoderm quality, and implantation [2]. In a prospective, blinded, multi-centric study we confirmed that the cf-mtDNA/cf-gDNA ratio in day 3 spent culture medium, combined with embryo morphology, improved the prediction upon blastulation compared to morphology alone, without any influence from patient and treatment-related factors [3].

Our opinion is that the cf-mtDNA in the embryo spent culture media would inversely mirror the overall embryonic mtDNA content, in line with recent studies suggesting that mtDNA abundance within blastomers may be correlated to aneuploidy, low embryo viability and development [4-6]. Undoubtedly, a direct comparison of mtDNA content between embryo and medium would provide a definitive demonstration of this hypothesis. The first criticism about the utility of cf-mtDNA as embryo biomarker was the potential genetic contamination from embryo-associated structures (i.e., cumulus cells, polar bodies or sperm) and human serum albumin supplement of the culture medium [7]. In reality, the levels of cf-mtDNA were considerably higher for media co-cultured with embryos respect to the low baseline level of control samples $[7,8]$. Because of mtDNA has a cytoplasmatic localization, the developing embryo may release it into the culture media through mechanisms that are independent of the nuclear DNA.

It is reasonable that the cf-mtDNA could be generated from dead or fragmented blastomeres-that's why higher cf-mtDNA was associated with increased levels of day 2-3 embryo fragmentation [1] or actively secreted throughout pre-implantation development in a stage-specifically way - here because cf-mtDNA increased from the cleavage to the blastocyst stage and the highest amount of cf-mtDNA was found in continuous single-culture media [7]. Such a stage-specifically mtDNA release by embryos could further explain the discrepant conclusions reported on the basis of which stage of embryo development the cf-mtDNA was evaluated $[9,10]$. We believe that cf-mtDNA could be a novel, non-invasive, easy accessible, molecular-based marker of optimal development potential, increasing the productivity of the merely morphological analysis on which embryo evaluation is traditionally based. Nevertheless, we are aware that some questions are still open and need to be answered to validate cf-mtDNA as a reliable predictor in clinical practice:

a. $\quad$ To uniquely define at what timing (i.e., on day 3, day 5) the cf-mtDNA quantification should be performed to be used as a biomarker, in combination with embryo morphology/ morphokinetics parameters. 
b. To identify for which embryological/clinical outcome (i.e., blastulation, euploidy, implantation) the combined morphology-cf-mtDNA data could be useful.

c. To optimize the best methodological approach, concerning the most appropriate target gene(s) to represent the copy number of cf-mtDNA and the most sensitive and specific technique (i.e., quantitative-PCR or digital PCR).

For this purposes, randomized controlled studies should be performed on large set of samples applying robust univariable and multivariable analyses and complex statistical methodologies, i.e., a generalized estimating equation model to consider the issue of subsequent cycles in the same woman, embryos from the same woman, etc.

\section{References}

1. Stigliani S, Anserini P, Venturini PL, Scaruffi P (2013) Mitochondrial DNA content in embryo culture medium is significantly associated with human embryo fragmentation. Hum Reprod 28(10): 2652-2660.

2. Stigliani S, Persico L, Lagazio C, Anserini P, Venturini PL, et al. (2014) Mitochondrial DNA in day 3 embryo culture medium is a novel, noninvasive biomarker of blastocyst potential and implantation outcome. Mol Hum Reprod 20(12): 1238-1246.

3. Stigliani S, Orlando G, Massarotti C, Casciano I, Bovis F, et al. (2019) Noninvasive mitochondrial DNA quantification on day 3 predicts blastocyst development: A prospective, blinded, multicentric study. Mol Hum Reprod 25(9): 527-537.
4. Juan DA, Rubio C, Marin C, Martinez S, Asmar AN, et al. (2015) Mitochondrial DNA content as a viability score in human euploid embryos: less is better. Fertil Steril 104(3): 534-541.

5. Fragouli E, Spath K, Alfarawati S, Kaper F, Craig A, et al. (2015) Altered levels of mitochondrial DNA are associated with female age, aneuploidy, and provide an independent measure of embryonic implantation potential. PLoS Genet 11: e1005241.

6. Ravichandran K, McCaffrey C, Grifo J, Morales A, Perloe M, et al. (2017) Mitochondrial DNA quantification as a tool for embryo viability assessment: Retrospective analysis of data from single euploid blastocysts transfers. Hum Reprod 32(6): 1282-1292.

7. Hammond ER, McGillivray BC, Wicker SM, Peek JC, Shelling AN, et al. (2017) Characterizing nuclear and mitochondrial DNA in spent embryo culture media: Genetic contamination identified. Fertil Steril 107(1): 220-228.

8. Rodriguez VM, Juan DA, Almazan JJ, Martinez S, Navarro R, et al. (2018) Origin and composition of cell-free DNA in spent medium from human embryo culture during preimplantation development. Hum Reprod 33(4): 745-756.

9. Zhang X, Sun Y, Dong X, Zhou J, Sun F, et al. (2019) Mitochondrial DNA and genomic DNA ratio in embryo culture medium is not a reliable predictor for in vitro fertilization outcome. Sci Rep 9: 5378.

10. Kobayashi M, Kobayashi J, Shirasuna K, Iwata H (2020) Abundance of cell-free mitochondrial DNA in spent culture medium associated with morphokinetics and blastocyst collapse of expanded blastocysts. Reprod Med Biol 19(4): 404-414.

For possible submissions Click below: 